\title{
Doppler cooling of an optically dense cloud of trapped atoms
}

\author{
Piet O. Schmidt, Sven Hensler, Jörg Werner, Thomas Binhammer, Axel Görlitz, and Tilman Pfau \\ 5. Physikalisches Institut, Universität Stuttgart, Pfaffenwaldring 57, D-70550 Stuttgart
}

\begin{abstract}
We have studied a general technique for laser cooling a cloud of polarized trapped atoms down to the Doppler temperature. A one-dimensional optical molasses using polarized light cools the axial motional degree of freedom of the atoms in the trap. Cooling of the radial degrees of freedom can be modelled by reabsorption of scattered photons in the optically dense cloud. We present experimental results for a cloud of chromium atoms in a magnetic trap. A simple model based on rate equations shows quantitative agreement with the experimental results. This scheme allows us to readily prepare a dense cloud of atoms in a magnetic trap with ideal starting conditions for evaporative cooling.
\end{abstract}

PACS numbers: $020.4180,020.7010,140.3320$

\section{INTRODUCTION}

Since the advent of laser cooling of neutral atoms more than 20 years ago, this method has been extensively studied [1] and widely used for a variety of atoms and applications. Ultimately, laser cooling followed by evaporative cooling [2] allowed the creation of a Bose-Einstein condensate (BEC) of neutral atoms [3, 4, 5].

In almost all BEC experiments atoms are caught in a magneto-optical trap. After sub-Doppler molasses cooling [6] and polarization of the sample by optical pumping, the atoms are typically transferred into a magnetic trap [3, 4, 5] or, as has been recently demonstrated, into an optical [7] trap. The consecutive evaporative cooling process requires both, a high initial density to provide a reasonably large elastic collision rate, and a large number of atoms, since most atoms are removed from the trap during evaporation. The figure of merit for efficient evaporative cooling is the initial phase space density of the atomic cloud. Therefore, all laser cooling and polarization steps have to be optimized carefully, to keep the temperature of the atoms low and the number of atoms high, thus maximizing the phase space density in the final trap.

In this paper, we present a robust Doppler cooling scheme in an external trap which is applicable to most laser-coolable atomic species. Our scheme is particularly well suited for optically dense samples and reduces the figure of merit for evaporative cooling to the number of atoms transferred into the trap regardless of temperature.

Free-space one-dimensional Doppler cooling is usually performed in a standing wave light field created by two counterpropagating laser beams with a frequency below the atomic transition frequency. Cooling is based on preferential absorption of photons from the laser beam opposing the direction of motion of the atom. Subsequent spontaneous emission is centrally symmetric and does not change the mean momentum of the atom. Doppler cooling of polarized atoms in a magnetic trap has been proposed for atomic hydrogen [8] and experimentally realized for sodium [9], hydrogen [10] and lithium [11]. In
Ref. 9 one-dimensional Doppler cooling of an optically thin cloud of sodium atoms in a magnetic trap was performed. The atoms were treated as a two level system since a very high magnetic offset field $\left(B_{0} \approx 1500 \mathrm{G}\right)$ spectroscopically resolved the Zeeman substates. Cooling of the motional degrees of freedom orthogonal to the laser beams was provided by anharmonic mixing in the trapping potential. Due to a long mixing time compared to the cooling time, the achieved temperature was ten times the Doppler temperature for sodium. In Ref. 10 single-beam pulsed Doppler cooling was performed on a dense sample of magnetically trapped hydrogen atoms. Thermalization of the cloud was accomplished by elastic collisions between the atoms. Cooling was limited to approx. five times the Doppler temperature due to limited laser power and additional heating rates.

The main advantage of the cooling technique presented here is that it combines three-dimensional temperatures close to the Doppler-limit with the cooling of dense samples. In our experiment, one-dimensional optical molasses cools the cloud of atoms in axial direction down to the Doppler limit [1]. Cooling in the radial directions, orthogonal to the laser beams, can be explained by reabsorption of spontaneously emitted photons by the optically dense cloud. To our knowledge, reabsorption has so far been treated only as a density-limiting mechanism which comes along with heating of the atomic sample 112, 13, 14, 15]. Here, we focus on the cooling aspects of scattered and reabsorbed photons in a trapped polarized atomic sample.

We show that the atoms in the magnetic trap remain polarized during the cooling process and practically no atoms are lost, provided the magnetic substructure is spectrally resolved. We are able to cool chromium atoms in a magnetic trap from $\approx 1 \mathrm{mK}$ to a mean temperature of $240 \mu \mathrm{K}$, corresponding to an increase in phase space density by a factor of 80 . We have studied the dynamics of the cooling process as well as the steady state temperature for various cooling parameters. The experimental results can be explained with a simple model based on rate equations for the temperature.

The paper is organized as follows. In Sec. 2, we present a theoretical model for Doppler cooling in a magnetic 
trap in the presence of reabsorption. The setup and experimental techniques for our cooling experiments with chromium are described in Sec. 3. Results on the dynamics and steady state properties of the cooling process are presented in Sec. 4. We conclude with a discussion of our results in Sec. 5 .

\section{THEORY}

\section{A. Rate Equations}

In this section, we present a simple model based on rate equations. Although we assume cooling in a magnetic trap here, the scheme is universal to all kinds of traps, provided cooling is compatible with the trap and the quantization axis of the atoms is independent of the position of the atoms in the trap.

Consider a cloud of spin-polarized atoms with mass $m$ and magnetic moment $\mu$ confined in an axially symmetric harmonic magnetic trap with trapping potential $V(x, y, z)=\mu\left(\frac{1}{2} B_{x}^{\prime \prime} x^{2}+\frac{1}{2} B_{y}^{\prime \prime} y^{2}+\frac{1}{2} B_{z}^{\prime \prime} z^{2}+B_{0}\right)$.

$B_{x, y, z}^{\prime \prime}$ are the magnetic field curvatures in radial $(x$, $y$ ) and axial $(z)$ direction, respectively, and $B_{0}$ is a homogeneous offset field along the axial direction. We assume that the corresponding trap frequencies $\omega_{x, y, z}=$ $\sqrt{\frac{\mu B_{x, y, z}^{\prime \prime}}{m}}$ are much smaller than the Larmor precession frequency at the center of the trap $\omega_{L}=\frac{\mu B_{0}}{\hbar}$, ensuring that the atoms stay spin polarized and no spin flip losses occur [16].

Doppler cooling is performed on an electric dipole transition that couples a long-lived ground state with total angular momentum $J$ to a short-lived excited state with angular momentum $J^{\prime}$, such that $J^{\prime}=J+1$ [17]. We also assume the corresponding Landé factors $g_{J}$ and $g_{J^{\prime}}$ to have the same sign, which we choose to be positive for our example. The atoms are polarized in the "weak field seeking" state $m_{J}=J$ and have a magnetic moment of $\mu=m_{J} g_{J} \mu_{B}$, where $\mu_{B}$ is Bohr's magneton.

Cooling is carried out in a $\sigma^{+} / \sigma^{+}$-standing wave created by two laser beams propagating along the axial direction with normalized intensity $I=I_{\text {laser }} / I_{\text {sat }}$ and detuning $\Delta=\left(\omega_{\text {laser }}-\omega_{\text {atom }}\right) / \Gamma$ from the unperturbed atomic transition frequency. $I_{\text {sat }}$ and $\Gamma$ are the saturation intensity and the linewidth of the transition, respectively.

In general, the quality of the polarization of the cooling light is important. For perfectly polarized $\sigma^{+}$-light propagating along the quantization axis of the atoms in a homogeneous magnetic field, no depolarizing transitions $m_{J} \longrightarrow m_{J}, m_{J}-1$ can occur. This is also true for excitation by scattered photons since the polarization with respect to the quantization axis of the incident light is preserved in the scattering process if the propagation direction coincides with the orientation of the magnetic field. For imperfect polarization, the cooling light can also drive depolarizing transitions which cause loss of atoms. In the configuration described above, the

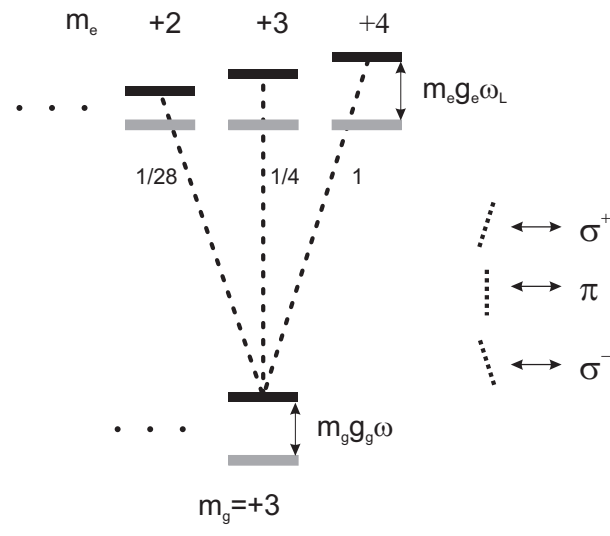

FIG. 1: Part of the Zeeman substructure of ${ }^{52} \mathrm{Cr}$ as an example of a $J \longrightarrow J+1$ transition. Shown is the transition from the electronic ground state ${ }^{7} S_{3}$ to ${ }^{7} P_{4}$ with a wavelength of $\lambda_{\text {atom }}=425.55 \mathrm{~nm}$, a saturation intensity $I_{s}=85.2 \mathrm{~W} / \mathrm{m}^{2}$ and a linewidth of $\Gamma=2 \pi \times 5.02 \mathrm{MHz}$. The numbers next to the transitions are the squares of the Clebsch-Gordan coefficients.

Zeeman-splitting due to the finite offset field $B_{0}$ additionally reduces the scattering rates $R_{\text {dep }}$ of unwanted depolarizing transitions with respect to the rate $R_{\mathrm{pol}}$ of the cooling transition $m_{J} \rightarrow m_{J}+1$ :

$$
R_{\mathrm{dep}}=\frac{\Gamma}{2} \frac{2 I C_{3}^{3}}{1+4 \Delta_{d e p}^{2}} \ll \frac{\Gamma}{2} \frac{2 I C_{3}^{4}}{1+4 \Delta_{\text {pol }}^{2}}=R_{\mathrm{pol}},
$$

where $\Delta_{\text {pol }}$ and $\Delta_{\text {dep }}$ are the effective detunings between the laser and the atomic transition frequency in a magnetic field and $C_{i}^{j}$ is the square of the Clebsch-Gordan coefficient for the transition from state $m_{g}=i$ to $m_{e}=j$. In Fig. 1 this effect is illustrated for the $J=3 \longrightarrow J=4$ transition that connects the ground state ${ }^{7} S_{3}$ to the excited state ${ }^{7} P_{4}$ in ${ }^{52} \mathrm{Cr}$. A full treatment of optical pumping in an arbitrary light field taking into account all excited and ground state levels gives the steady state population of each ground state for a given magnetic field [18]. Assuming a polarization quality of 1:10, a detuning of $\Delta=-0.5$ for the polarizing transition and allowing for an atom loss of less than $1 \%$ during the cooling process, we find for the case of chromium a necessary offset field of $B_{0} \geq 5 \mathrm{G}$ and for rubidium $B_{0} \geq 15 \mathrm{G}$.

Following the common treatment of free-space Doppler cooling [1, 9], rate equations for the temporal evolution of the temperature for the axial $(z)$ and radial $(y)$ degrees of freedom are readily obtained:

$$
\begin{aligned}
& \frac{d T_{z}}{d t}=\frac{E_{R} k_{B}}{2 \tau_{z}^{\text {heat }}}-\frac{T_{z}}{\tau_{z}^{\text {cool }}} \\
& \frac{d T_{y}}{d t}=\frac{E_{R} k_{B}}{2 \tau_{y}^{\text {heat }}}-\frac{T_{y}}{\tau_{y}^{\text {cool }}},
\end{aligned}
$$

where $E_{R}=\frac{\hbar^{2} k^{2}}{2 m}$ is the recoil energy of the cooling transition with wavenumber $k=2 \pi / \lambda$ and $k_{B}$ is the Boltzmann 
constant. Usually, the heating and cooling time constants are derived by considering the random walk in momentum space due to spontaneous emission and the net cooling effect of scattered photons from the two counterpropagating laser beams [1]. In an optically dense sample reabsorption of photons has to be taken into account. Previously, reabsorption has mainly been discussed as a reason for density limitations and density-dependent heating in magneto-optical traps 112, 13, 14 and in the context of sub-recoil cooling [15]. In our model, we emphasize cooling effects of reabsorbed photons which can be substantial for polarized atoms confined in an external trapping potential.

The photon energy after a scattering event in the low intensity limit [19] is shifted by at most $4 E_{R}+2 \hbar k v \ll$ $\hbar\left|\Delta_{\text {pol }}\right| \Gamma$, where $v$ is the velocity of an atom at Doppler temperature. Therefore scattered photons have essentially the same detuning and polarization as the cooling laser beams. For our calculations, we use the axial direction parallel to the laser beam as our quantization axis. The contribution of the reabsorbed photons to cooling of the radial and axial directions depends on the projection of their wave vector $\vec{k}$ onto these directions. The effective intensity $I_{z, y}^{\mathrm{eff}}$ of the cooling photons is proportional to the incident laser light intensity $2 I$ with a proportionality factor $\kappa_{z, y}$ which primarily depends on the optical density $O D_{z, y}$ in these directions:

$$
I_{z, y}^{\mathrm{eff}}=2 I \times \kappa_{z, y} \sim 2 I \times \kappa_{z, y}^{*} \times O D_{z, y}
$$

If we consider a dipole radiation and absorption pattern for $\sigma$-polarized light and take into account the $1 / e^{2}$ size $\sigma(\theta)$ of a Gaussian shaped cloud in the direction given by $\theta$, we arrive at the following expressions:

$$
\begin{aligned}
& \kappa_{y}=\kappa_{0} \frac{2}{\pi} \int_{0}^{\pi} d \theta\left(1+\cos ^{2} \theta\right)^{2} \sigma(\theta) \sin ^{2} \theta \\
& \kappa_{z}=\kappa_{0} \int_{0}^{\pi} d \theta\left(1+\cos ^{2} \theta\right)^{2} \sigma(\theta) \sin \theta|\cos \theta|
\end{aligned}
$$

$\kappa_{0}=n_{0} \tilde{\sigma}_{\lambda} \frac{9}{128 \sqrt{2 \pi}}$ is an angle-independent constant, with peak density $n_{0}$ and resonant absorption cross section $\tilde{\sigma}_{\lambda}=6 \pi \lambda^{2}$ which we assume to be uniform over the cloud. In a simple picture, $\kappa_{z, y}$ can be interpreted as the effective number of photons that contribute to cooling in the corresponding directions originating from a single photon absorbed from the laser light. The total number of reabsorption/emission cycles for an incident photon is given by

$$
\kappa=\kappa_{0} \int_{0}^{\pi} d \theta\left(1+\cos ^{2} \theta\right)^{2} \sigma(\theta) \sin \theta .
$$

If the effects of reabsorbed photons are included the cool- ing rates become 9

$$
\begin{aligned}
\frac{1}{\tau_{z}^{\text {cool }}} & =-\Gamma \frac{32 \Delta_{\mathrm{pol}}\left(1+\kappa_{z}\right) 2 I}{\left(1+4 \Delta_{\mathrm{pol}}^{2}\right)^{2}} \frac{E_{R}}{\hbar \Gamma} \\
\frac{1}{\tau_{y}^{\text {cool }}} & =-\Gamma \frac{32 \Delta_{\mathrm{pol}} \kappa_{y} 2 I}{\left(1+4 \Delta_{\mathrm{pol}}^{2}\right)^{2}} \frac{E_{R}}{\hbar \Gamma} .
\end{aligned}
$$

In axial and radial direction the total cooling light intensity reads $I_{z}^{\text {tot }}=2 I+I_{z}^{v}=2 I\left(1+\kappa_{z}\right)$ and $I_{y}^{\text {tot }}=I_{y}^{v}=$ $2 I \kappa_{y}$, respectively.

For the derivation of the heating rates, we have to take into account directed reabsorption of $\kappa_{z, y}$ photons and spontaneous emission of $(\kappa+1)$ photons for each photon absorbed from the cooling laser beams:

$$
\begin{aligned}
\frac{1}{\tau_{z}^{\text {heat }}} & =\left[\left(1+\kappa_{z}\right)+\frac{2}{5}(1+\kappa)\right] \frac{2 I \Gamma}{1+4 \Delta_{\text {pol }}^{2}} \\
\frac{1}{\tau_{y}^{\text {heat }}} & =\left[\kappa_{y}+\frac{3}{10}(1+\kappa)\right] \frac{2 I \Gamma}{1+4 \Delta_{\text {pol }}^{2}}
\end{aligned}
$$

The factors $2 / 5$ and $3 / 10$ are statistical weights from the dipole radiation pattern for the different directions of spontaneous emission. In this model we have neglected additional loss and heating processes, like e.g. radiative escape [20] and radiation trapping [12]. The influence of these additional effects will be discussed in the experimental part of this paper.

\section{B. Discussion of the Model}

\section{Steady State}

For simplicity we assume the coefficients $\kappa_{z, y}$ to be time independent. This is justified for the evaluation of the steady state parameters of the cooling process. The dynamics of the cooling process can be described only qualitatively within this model, since the optical density will change during cooling and therefore the number of reabsorbed photons increases.

The steady state temperatures are readily derived from Eqns. 2, 3 to be

$$
\begin{aligned}
& T_{z}^{\infty}=\frac{\tau_{z}^{\text {cool }}}{\tau_{z}^{\text {heat }}} \frac{E_{R} k_{B}}{2}=\frac{1+\kappa_{z}+\frac{2}{5}(1+\kappa)}{1+\kappa_{z}} \frac{T_{D}}{2} \\
& T_{y}^{\infty}=\frac{\tau_{y}^{\text {cool }}}{\tau_{y}^{\text {heat }}} \frac{E_{R} k_{B}}{2}=\frac{\kappa_{y}+\frac{3}{10}(1+\kappa)}{\kappa_{y}} \frac{T_{D}}{2},
\end{aligned}
$$

where $T_{D}=\frac{\hbar \Gamma}{2 k_{B}}$ is the Doppler temperature. These minimum temperatures are found at a detuning of $\Delta_{\mathrm{pol}}=$ $-\frac{1}{2}$, in agreement with free space Doppler cooling [1]. It is worthwhile to mention that in this configuration in absence of reabsorption (i.e. $\kappa_{z, y}=\kappa=0$ ) the $1 \mathrm{D}$ temperature in axial direction

$$
T_{z}^{\min }=\frac{7}{10} T_{D}
$$




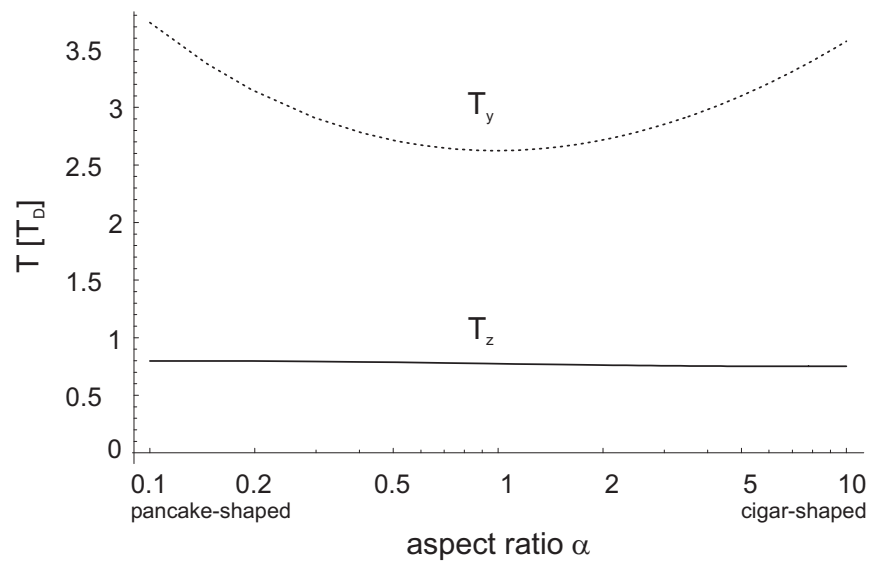

FIG. 2: Temperatures in axial $(z)$ and radial $(y)$ direction as a function of trap aspect ratio for $N=10^{8}$ atoms a peak density of $n_{0}=5 \times 10^{10} \mathrm{~cm}^{-3}$.

is smaller than what is usually called the 1D Doppler temperature. The reason is easily seen: in the 1D model photons can be absorbed and emitted only along the axial direction; in a 3D model with $1 \mathrm{D}$ cooling, photons can be emitted into radial degrees of freedom according to the dipol radiation pattern. These degrees of freedom take up photon recoil heating and therefore reduce the heating rate in the axial direction. In this situation the radial energy grows without limit.

If we now include reabsorption, the situation changes. Whereas reabsorption only in axial direction $\left(\kappa_{z}>0\right)$ changes the cooling rate but not the steady state temperature, reabsorption in radial direction $\left(\kappa_{y}>0\right)$ provides a cooling effect, that establishes a radial steady state temperature (Eq. 14). The magnitude of this temperature is determined by the ratio of heating to cooling photons.

Experimentally, it is not possible to independently change $\kappa_{y}, \kappa_{z}$ and $\kappa$. They depend via Eqns. 7 and 8 on the shape of the trapped cloud. In Fig. 2 we show an example, where we have integrated these equations for different aspect ratios $\alpha=\sigma_{z} / \sigma_{y}$ to calculate the steady state temperatures. The aspect ratio has no influence on the axial temperature, since cooling is dominated by photons scattered directly from the laser beams. In radial direction we observe a minimum near a spherical cloud. For a cigar shaped cloud the optical density in radial direction decreases and for a pancake-shaped cloud the solid angle decreases in which photons contributing to radial cooling can be scattered.

\section{Dynamics}

The transient evolution of the temperature in radial and axial direction can be approximated by the well known solution of Eqns. 2, 3:

$$
T_{z, y}(t)=T_{z, y}^{\infty}+\left(T_{z, y}^{0}-T_{z, y}^{\infty}\right) \exp \left(-\frac{t}{\tau_{z, y}^{c o o l}}\right)
$$

where $T_{z, y}^{0}$ are the initial temperatures of the cloud in axial and radial direction. Depending on the initial temperatures and the steady state temperatures Eqns. 13, 14, either Doppler cooling $\left(T_{z, y}^{\infty}<T_{z, y}^{0}\right)$ or Doppler heating $\left(T_{z, y}^{\infty}>T_{z, y}^{0}\right)$ occurs. However, since the atoms are oscillating in a harmonic trap, cooling preferentially occurs close to the trap minimum where the velocity of the atoms is highest. Therefore the cooling rates can not be higher than approx. one quarter of the trap oscillation period $t_{z, y}$.

According to Eqns. 9, 10 the time constants $\tau_{z}^{\text {cool }}$, $\tau_{y}^{\text {cool }}$ depend on the number of scattered cooling photons. This can provide a test for the reabsorption model. If cooling in radial direction originated from elastic scattering 10] or anharmonic mixing by the trapping potential [9], one would expect an intensity independent relaxation towards an equilibrium temperature, provided $\tau_{z}^{\text {cool }} \ll \tau_{y}^{\text {cool }}$, while our model explicitly requires an intensity dependence of $\tau_{y}^{\text {cool }}$

We have already mentioned in the previous section that the effective intensity of reabsorbed photons will change during the cooling process. We will now discuss the dynamical effects on the relevant parameters $\kappa_{z, y}$. From Fig. 2 and Eq. 13 we deduce a weak dependence of the axial steady state temperature on reabsorbed photons. In this regime cooling in axial direction is dominated by the real laser beams and we can assume $T_{z}$ to be unperturbed from its minimum value $T_{z}^{\min }$. On the contrary, radial cooling strongly depends on the reabsorbed photons, so we have to take into account how $\kappa_{y}$ changes with time. As cooling in the $z$-direction starts, the cloud shrinks according to $\sigma_{z}=\sqrt{\frac{k_{B} T_{z}}{\mu B_{z}^{\prime \prime}}}$. That will increase the optical density in radial direction and thus the number of reabsorbed photons according to Eq. 5 and

$$
O D_{y} \propto \frac{1}{\sigma_{z} \sigma_{y}} \propto \kappa_{y} .
$$

As a result, the radial steady state temperature is reduced, since it depends on the effective number of reabsorbed photons (Eq. 14). The same argument holds for the size of the cloud in radial direction. During radial cooling $\sigma_{y}$ shrinks and we arrive at an even lower $T_{y}^{\infty}$. In actual experiments this nonlinear effect is less pronounced and will be neglected.

\section{EXPERIMENTAL SETUP}

In our experiment, we continuously load typically $10^{8}$ chromium atoms in the low field seeking $\mid j=3, m_{J}=$ $3>$ magnetic substate into a weak Ioffe-Pritchard trap [21] in the cloverleaf configuration [22]. Details of the continuous loading mechanism can be found in Refs. 23, 24. In the compressed cigar-shaped trap with a nearly harmonic trapping potential in all three dimensions, we have an axial and a radial curvature of $B_{z}^{\prime \prime}=110 \mathrm{G} / \mathrm{cm}^{2}$ and $B_{y}^{\prime \prime}=750 \mathrm{G} / \mathrm{cm}^{2}$, respectively. This corresponds 
to trapping frequencies for chromium $\mu=6 \mu_{B}$ of $\omega_{x}=$ $\omega_{y}=2 \pi \times 110 \mathrm{~Hz}$ and $\omega_{z}=2 \pi \times 42 \mathrm{~Hz}$. We use a rather high offset field of $B_{0}=28 \mathrm{G}$ to assure sufficient harmonicity in all directions. During compression the atoms are adiabatically heated to a temperature of $1 \mathrm{mK}$ at a peak density of $0.4-1 \times 10^{10} \mathrm{~cm}^{-3}$. In this trap we perform one-dimensional Doppler cooling with a retroreflected $\sigma^{+}$-polarized beam along the axial direction of the trap. Laser light at $425.55 \mathrm{~nm}$ from a frequency doubled Ti:Sapphire laser drives the ${ }^{7} \mathrm{~S}_{3} \longrightarrow{ }^{7} \mathrm{P}_{4}$ transition in ${ }^{52} \mathrm{Cr}\left(\Gamma=2 \pi \times 5.02 \mathrm{MHz}, I_{\text {sat }}=85.2 \mathrm{~W} / \mathrm{m}^{2}\right.$, see Fig. 11). The laser frequency is tuned to $7 \Gamma$ above the unperturbed transition frequency corresponding to an effective detuning of approx. $-0.8 \Gamma$ for a nonmoving atom placed at the minimum of the magnetic field in the center of the trap. This value was optimized with respect to minimum temperature in all directions. The mean intensity of the approx. $1 \mathrm{~cm}$ diameter beam as seen by the approx. $2 \mathrm{~mm}$ diameter cloud was measured to be $4 \times 10^{-3} I_{\text {sat }}$ using a $1 \mathrm{~mm}$ diameter pinhole in front of a calibrated photodiode unless otherwise noted. The atomic ensemble is detected by absorption imaging using a 12 Bit CCD camera. The resulting optical density profile of the cloud is fitted with two orthogonal Gaussian profiles meeting at the center of the cloud. The number of atoms is extracted from this fit using the peak optical density and the size. We perform time-of-flight (TOF) sequences to obtain the temperature in axial and radial direction. Densities are derived using the size of the cloud in the trap obtained from the time-of-flight fit and the number of atoms. The error bars shown in the figures are the square root of the diagonal elements of the covariance matrix for the fitting parameters obtained from a least square fit. No systematic errors have been included, unless otherwise noted.

\section{EXPERIMENTAL RESULTS}

\section{A. Dynamics}

In this section, we present experimental results on the dynamics of the cooling process. In Fig. 3 we have plotted the temperature of the cloud in axial $(z)$ and radial (y) direction after a variable cooling time. We start in both directions with a temperature of approx. $1 \mathrm{mK}$. In axial (cooling laser beam) direction we see a fast decrease in temperature. But also in the radial direction, where no laser beam is applied, cooling is observed on a short timescale. The initial decrease in temperature is very well fitted by an exponential decay in both cases, as is expected from Eq. 16. The cooling time constants derived from the fit are $\tau_{z}^{\text {cool }} \approx 10 \mathrm{~ms}$, corresponding to the fastest possible cooling time of $\frac{2 \pi}{4 \omega_{z}}$ and $\tau_{y}^{\text {cool }} \approx 50 \mathrm{~ms}$. In fact, we have never observed axial cooling time constants significantly lower than $10 \mathrm{~ms}$. The minimum steady state temperatures in Fig. 3 are $T_{y}^{\infty}=334 \pm 7 \mu \mathrm{K}$ and $T_{z}^{\infty}=124 \pm 3 \mu \mathrm{K}$, corresponding to 2.7 and 1 times the $1 \mathrm{D}$ Doppler temperature of $T_{D}=124 \mu \mathrm{K}$. Due to den-

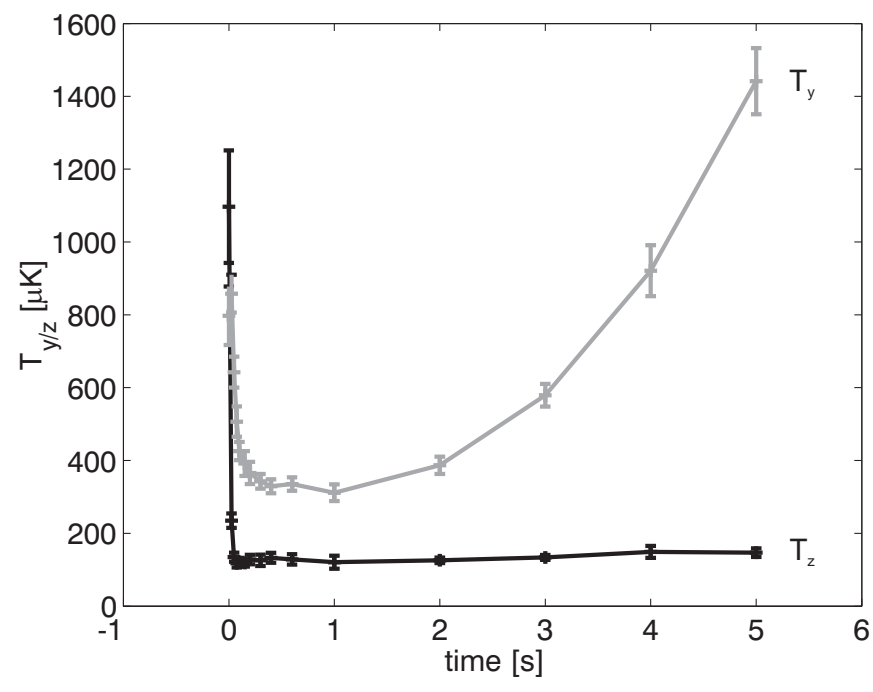

FIG. 3: Typical evolution of the temperatures in axial $(z)$ and radial $(y)$ direction as a function of cooling time. The intensity of the cooling laser was $4 \times 10^{-3} I_{\text {sat }}$.

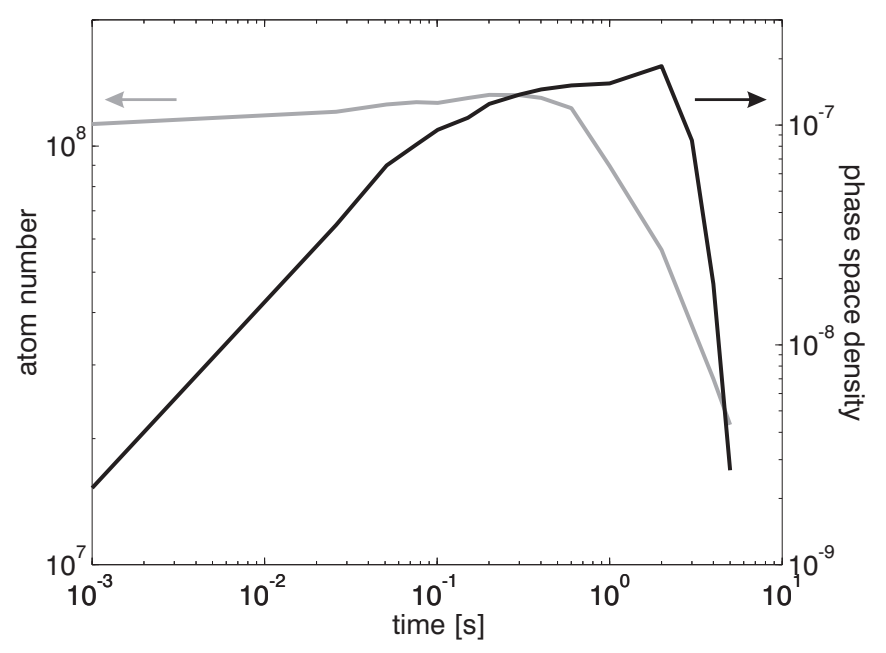

FIG. 4: Evolution of the number of atoms and the phase space density for the same data set as shown in Fig. 3 .

sity dependent heating effects in the light field [25, 26], the minimum axial temperature of $T_{z}^{\text {min }} \approx 90 \mu \mathrm{K}$ is not reached. Fig. I shows the evolution of the number of atoms and the phase space density during the cooling in a double logarithmic plot to resolve the fast initial dynamics. The number of atoms stays constant within experimental uncertainty beyond $300 \mathrm{~ms}$ at which time the steady state temperature is reached. We gain more than a factor of 80 in phase space density and increase at the same time the peak density by more than one order of magnitude from $0.4 \times 10^{10}$ to $5.6 \times 10^{10} \mathrm{~cm}^{-3}$.

In the experiment shown in Figs. 3 and 1 , we continued cooling after reaching steady state. Fig. 1 shows a strong reduction in the number of atoms for cooling times longer than $500 \mathrm{~ms}$. Atoms are lost from the trap presumably by radiative escape or fine structure changing 


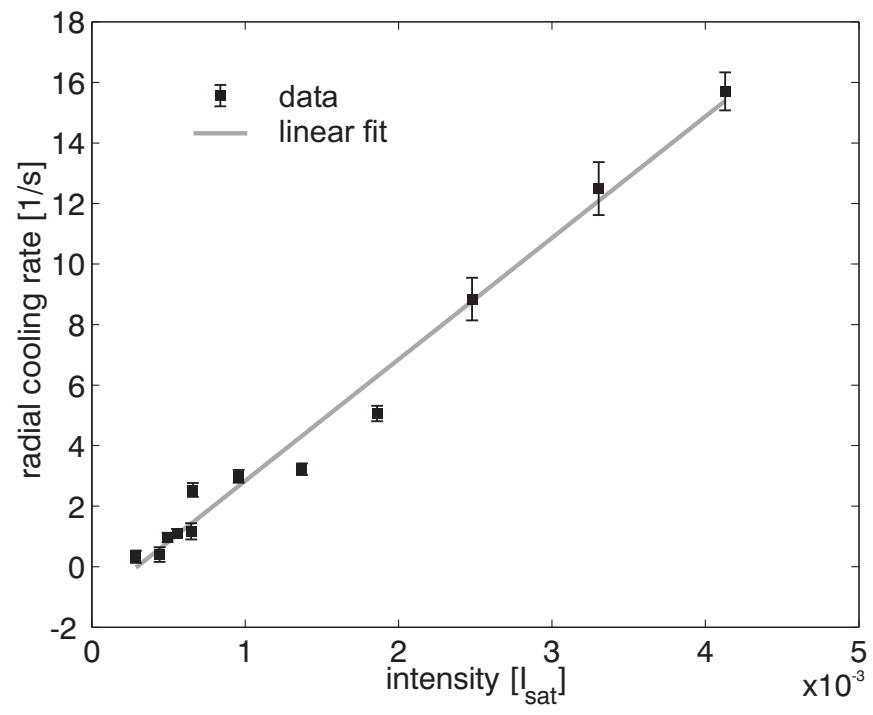

FIG. 5: Radial cooling rate $\frac{1}{\tau_{y}^{\text {coot }}}$ as a function of measured single beam cooling light intensity. The corresponding cooling rates in axial direction are larger by a factor of at least 5 for each data point.

collisions 20, 23 in the cooling light. At the same time the temperature increases in radial direction, whereas it stays constant in axial direction. This is evidence for a density dependent cooling effect in radial direction. A lower number of atoms results in a reduced optical density and, according to Eq. 14, to a higher steady state temperature $T_{y}^{\infty}$. We will come back to this effect in the discussion of the steady state results.

We have already pointed out in section $2 B$ that the cooling rate of the radial degrees of freedom via anharmonic mixing or elastic collisions between the atoms should be independent of cooling light intensity, provided cooling in axial direction is much faster. In Fig. 5 the results of a cooling experiment with different cooling light intensities are plotted. For each data point we have performed an experiment analogous to Fig. 3 and fitted the initial exponential decay of the temperature to obtain the cooling rate. The corresponding rate constant for the axial direction has been verified to be larger by at least a factor of five for each data point. One can clearly see a linear dependence of the radial cooling rate on light intensity, ruling out anharmonic mixing or elastic collisions as the major cooling mechanism. We have also performed an experiment in which we stopped cooling after the axial direction reached steady state, thus producing a highly anisotropic temperature distribution. The subsequent cross-dimensional relaxation of the temperatures driven by elastic ground state collisions occurred on a much longer timescale than the observed cooling time constants when the light was on.

Increasing the laser intensity beyond the values shown in Fig. 5 results in a strong distortion of the cloud due to light pressure forces and intensity imbalance between the two counterpropagating cooling beams. This causes

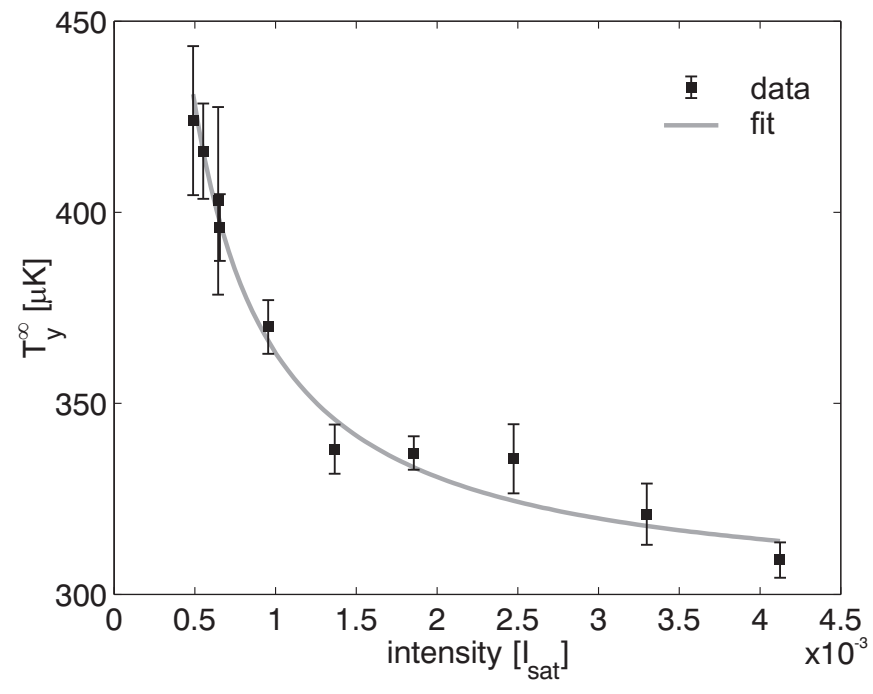

FIG. 6: Radial steady state temperature as a function of cooling light intensity. The data is fitted by Eq. 17. The data is from the same experimental run as in Fig.

parasitic heating and increases the achievable temperatures.

From the slope of the linear fit to the data in Fig. 5 we can extract $\kappa_{y}$ according to Eq. 10. Taking into account that the average intensity incident on an atom is a factor of 3 lower than the measured intensity due to absorption, we obtain $\kappa_{y}=0.023$ for a detuning of $\Delta_{\text {pol }}=-0.8$. To compare this experimentally determined value with theory, we have integrated Eq. 6 using the steady state size of the cloud $\sigma_{z}=700 \mu \mathrm{m}$ and $\sigma_{y}=410 \mu \mathrm{m}$. The theoretical result of $\kappa_{y}=0.1$ is larger by more than a factor 4. This deviation supposedly arises from uncertainties in the effective intensity as seen by the atoms. This includes a systematic error in the measured intensity, uncertainty in the averaged intensity due to absorption of photons from the laser beam and averaging effects for the detuning due to magnetic field curvature.

\section{B. Steady State Temperatures}

In this section, we present experiments that elucidate the different effects influencing the steady state temperature and compare them to theory.

In the simplified model used to derive the steady state temperatures in axial and radial direction given by Eqns. 13 and 14, the latter are independent of cooling light intensity, since both, the heating rate $1 / \tau_{z, y}^{\text {heat }}$ and the cooling rate $1 / \tau_{z, y}^{c o o l}$ are linear in intensity. Accounting for an additional constant heating rate $R$ in the differential equation (3), the steady state temperature for a detuning of $\delta=-0.5$ becomes

$$
T_{y}^{\infty}=\frac{\left(\kappa_{y}+\frac{3}{10}(1+\kappa)+\frac{R}{E_{R} \Gamma I}\right)}{\kappa_{y}} \frac{T_{D}}{2} .
$$




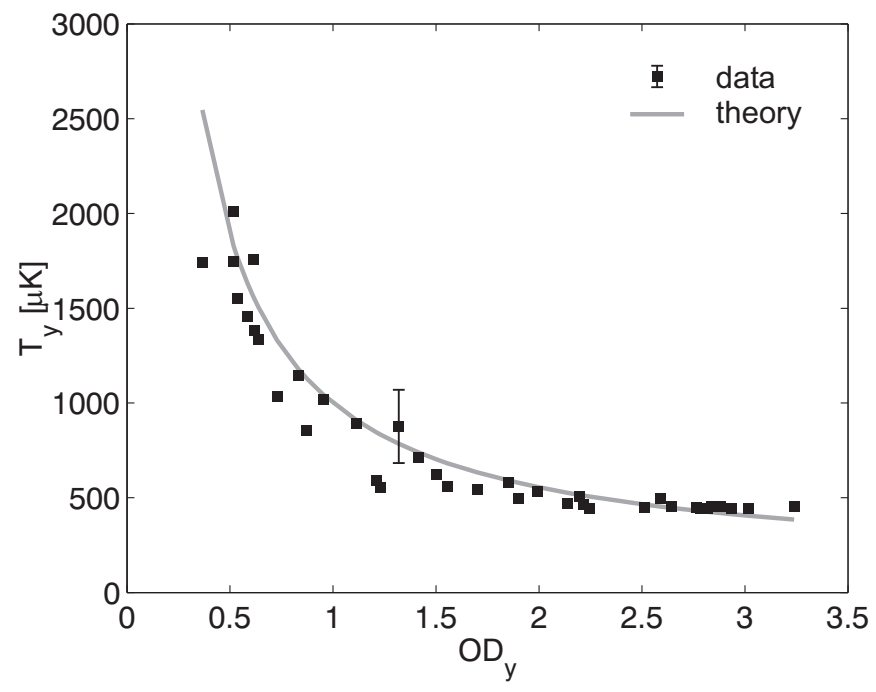

FIG. 7: Steady state temperature in radial direction as a function of optical density (OD) in this direction. The error bar resembles the typical error from the temperature fit to the time of flight data. The theory curve is a plot of Eq. 18 using the results of the fit from Fig. 6 .

In Fig. 6 we have plotted the measured steady state temperature $T_{y}^{\infty}$ for different cooling light intensities. The fit to Eq. 17 using the measured detuning of $\delta=-0.8$ and $\kappa=0.24$ calculated from Eq. 8, gives $\kappa_{y}=0.11 \pm 0.002$ and $R=2.6 \times 10^{-26} \pm 10 \% \mathrm{Js}^{-1}$. This result is in excellent agreement with the integrated value for $\kappa_{y}=0.1$ obtained from Eq. 6. From Fig. 6 we can also estimate a minimum radial temperature of $T_{y}^{\infty} \approx 300 \mu \mathrm{K}$ which is in good agreement with the predicted value using Eq. 14 and Fig. 2 of $T_{y}^{\infty}=2.3 T_{D} \approx 290 \mu \mathrm{K}$. Thus, the additional, intensity independent heating mechanism slightly increases the minimum temperature. We have identified this mechanism to be a strong dipolar relaxation process in chromium [27.

We have already seen in Fig. 3 that the steady state temperature in radial direction depends on the number of atoms in the cloud (increase in temperature for a decreasing number of atoms at long cooling times). To elucidate this effect more quantitatively we have performed an experiment in which we cool down the atoms to steady state as described before. In a second "cooling" stage we reduce the cooling laser intensity to $1.6 \times 10^{-3} I_{\text {sat }}$ to slow down the loss of atoms. After different cooling times, we measure the temperature and the optical density in radial direction. The result is plotted in Fig. 7 as a function of optical density in radial direction. We observe a decrease in temperature with increasing optical density. The effective number of reabsorbed radial and total cooling photons, $\kappa_{y}$ and $\kappa$, respectively, are to lowest order linearly proportional to the optical density $O D_{y}$ (Eq. 5). Introducing two new constants, $\kappa_{y}^{*}=\kappa_{y} / O D_{y}$ and $\kappa^{*}=\kappa / O D_{y}$, the steady state temperature reads

$$
T_{y}^{\infty}=\frac{\left(\kappa_{y}^{*} \times O D_{y}+\frac{3}{10}\left(1+\kappa^{*} \times O D_{y}\right)+\frac{R}{E_{R} \Gamma I}\right)}{\kappa_{y}^{*} \times O D_{y}} \frac{T_{D}}{2} .
$$

This equation is plotted as a solid line in Fig. 6 using the results for $R$ and $\kappa_{y}$ (normalized to steady state optical density of 4.5) from the fit to the data in Fig. 6. The agreement with the experimental data is surprisingly good, since $\kappa_{y}$ and $\kappa$ are not strictly linear in the optical density (see Eqns. 6, 8) and the heating rate depends on temperature and density.

\section{CONCLUSION}

We have presented a general Doppler cooling technique which works for optically dense, trapped atomic samples. In experiments with magnetically trapped Chromium atoms we have achieved an increase in phase space density by two orders of magnitude. To explain our experimental results, in particular the fast and efficient threedimensional cooling in a one-dimensional molasses, we have developed a model which takes into account radial cooling due to reabsorption of scattered photons. Our experimental findings for the intensity-dependence of the cooling rate and the steady-state temperature agree well with the theoretical considerations. We could exclude thermalization effects via elastic collisions or anharmonic mixing as the origin of the radial cooling we observe. Optimum radial cooling is observed in spherical trap configurations and the radial cooling efficiency increases with the radial optical density.

Our technique should be applicable to most atomic species which can be laser-cooled provided that a moderate magnetic offset-field (e.g. $B_{0}=15 \mathrm{G}$ for ${ }^{87} \mathrm{Rb}$ ) is applied to prevent depolarization of the sample. Implementation of this technique in a BEC experiment could simplify the initial laser cooling stages, since Doppler cooling in the trap reduces the figure of merit for loading of the trap to the number of atoms transferred into the trap regardless of temperature. Due to the low light intensities needed to cool the atoms, our method might also be applicable to magnetically trapped atoms in a cryogenic environment [28, 29]. In our experiment, the achieved phase space density for chromium already provides good starting conditions for evaporation and only excessive atom loss and heating due to dipolar relaxation [27 in the consecutive evaporation stage prevented us from reaching quantum degeneracy.

\section{Acknowledgments}

This work was funded by the Forschergruppe Quantengase der Deutschen Forschungsgemeinschaft. P.O.S 
has been supported by the Studienstiftung des deutschen Volkes.

[1] P. D. Lett, W. D. Phillips, S. L. Rolston, C. E. Tanner, R. N. Watts, and C. I. Westbrook, J. Opt. Soc. Am. B 6, 2084 (1989).

[2] W. Ketterle and N. van Druten, Adv. At. Mol. Opt. Phys. 37, 181 (1996).

[3] J. R. Anglin and W. Ketterle, Nature 416, 211 (2002).

[4] M. Inguscio, S. Stringari, and C. E. Wieman, eds., BoseEinstein Condensation in Atomic Gases (International School of Physics "Enrico Fermi", 1998).

[5] E. A. Cornell and C. E. Wieman, Rev. Mod. Phys 74 (2002).

[6] J. Dalibard and C. Cohen-Tannoudji, J. Opt. Soc. Am. B 6, 2023 (1989).

[7] M. Barrett, J. Sauer, and M. Chapman, Phys. Rev. Lett. 87, 010404 (2001).

[8] T. Hijmans, O. Luiten, I. Setija, and J. T. M. Walraven, J. Opt. Soc. Am. B 6, 2235 (1989).

[9] K. Helmerson, A. Martin, and D. E. Pritchard, J. Opt. Soc. Am. B 9, 1988 (1992).

[10] I. D. Setija, H. Werij, O. J. Luiten, M. W. Reynolds, T. W. Hijmans, and J. T. M. Walraven, Phys. Rev. Lett. 70, 2257 (1993).

[11] F. Schreck, G. Ferrari, K. L. Corwin, J. Cubizolles, L. Khaykovich, M.-O. Mewes, and C. Salomon, Phys. Rev. A 64, 011402 (2001).

[12] D. Sesko, T. Walker, and C. Wieman, J. Opt. Soc. Am. B 8, 946 (1991).

[13] G. Hillenbrand, C. J. Foot, and K. Burnett, Phys. Rev. A 50, 1479 (1994).

[14] K. Ellinger and J. Cooper, Phys. Rev. A 55, 4351 (1997).

[15] Y. Castin, J. Cirac, and M. Lewenstein, Phys. Rev. Lett.
80, 5305 (1998).

[16] C. Sukumar and D. Brink, Phys. Rev. A 56, 2451 (1997).

[17] In principle the same argument holds for a $J^{\prime}=J$ transition; for simplicity we will concentrate on the situation above.

[18] J. Stuhler, Dissertation, Universität Konstanz, Lehrstuhl J. Mlynek, Ufo-Verlag, Allensbach (2002).

[19] C. Cohen-Tannoudji, J. Dupont-Roc, and G. Grynberg, Atom-photon interactions (John Wiley \& Sons, 1992), 1st ed., page $93 \mathrm{ff}$.

[20] T. Walker and P. Feng, Advances in Atomic, Molecular, and Optical Physics 34, 125 (1994).

[21] T. Bergeman, G. Erez, and H. Metcalf, Phys. Rev. A 35, 1535 (1987).

[22] M.-O. Mewes, M. Andrews, N. van Druten, D. Kurn, D. Durfee, and W. Ketterle, Phys. Rev. Lett. 77, 416 (1996).

[23] J. Stuhler, P. O. Schmidt, S. Hensler, J. Werner, J. Mlynek, and T. Pfau, Phys. Rev. A 64, 031405 (2001).

[24] P. O. Schmidt and et al., Continuous loading of a ioffepritchard trap, in preparation.

[25] A. S. Bell, J. Stuhler, S. Locher, S. Hensler, J. Mlynek, and T. Pfau, Europhys. Lett. 45, 156 (1999).

[26] C. C. Bradley, J. J. McClelland, W. R. Anderson, and R. J. Celotta, Phys. Rev. A 61, 053407 (2000).

[27] P. O. Schmidt and et al., Dipolar relaxation in ultracold dipolar gases, in preparation.

[28] J. D. Weinstein, R. deCarvalho, C. I. Hancox, and J. M. Doyle, Phys. Rev. A 65, 021604 (2002).

[29] R. deCarvalho et al., same issue. 